\title{
IL CALCOLO DELLE SUPERFICI RIFLETTENTI NEI RILIEVI SISMICI A RIFLESSIONE
}

\author{
Camillo Contini
}

1. Premesse. - La velocita $v$ di trasmissione delle onde sismiche longitudinali in un mezzo elastico isotropo ed omogeneo ì data dalla

$$
v=\sqrt{\frac{E(1-n)}{\sigma(1+m)(1-2 m)}},
$$

ove $E$ ì il modulo di elasticita di Young, $m$ il rapporto di Poisson e $\sigma$ la densitá del mezzo stesso.

I valori delle costanti di elasticití e della densita dei terreni che costituiscono il sottosuolo sono funzione della compattezza e del grado di costipazione dei terreni stessi e quindi funzione delle pressioni cui questi sono sottrposti attualmente e furono sottoposti in passato, dei tempi d'azione delle pressioni stesse, delle temperature aqenti, dei fluidi contenuti e della loro costituzione e pressione, ecc. ( ${ }^{1}$.

Per le profonditi che interessano le ricerche minerarie, al massimo di una decina di chilometri, possiamo, con Gutenberg $(\stackrel{2}{ })$, porre uguale a $1 / 4$ il rapporto $m$ di Poisson, e quindi porre

$$
v=\sqrt{\frac{\sigma}{5} \frac{E}{\sigma}} \text {. }
$$

Se allora per i valori della densità e del modulo di elasticita adottiamo le formole seguenti, che si deducono da quelle di Schweydar,

$$
\begin{aligned}
& \sigma=\sigma_{v}\left(1+\alpha z+\beta z^{2}\right), \\
& e=E_{0}\left(1+\omega^{\prime} z+\beta^{\prime} z^{2}\right),
\end{aligned}
$$

ove $a, \hat{p}, u^{\prime}, \hat{p}$, sono delle costanti da determinare sperimentalmente e z la profondita, otteniamo per la velocita l'espressione

$$
v=v \sqrt{\frac{1+\alpha^{\prime} z+\beta^{\prime} z}{1+\alpha z+\bar{p} z^{z}}}
$$

essendo $v_{0}$ la velocità alla profonditì $z=0$. 
Se per la densita adottiamo la formola di Schweydar e per il modulo di elasticita adottiamo la formola di Prey

$$
E=E_{0}\left(1+a z+b z^{2}+c z^{3}+d z^{4}\right) \quad,
$$

ove $a, b, c, d$ sono al solito delle costanti da determinarsi sperimentalmente, abbiano

$$
v=v \sqrt{\frac{1+a z+b z^{2}+c z^{3}+d z^{4}}{1+\alpha z+h z^{2}}}
$$

Le determinazioni sperimentali della densita fatte su campioni prelevati a diverse profondità, mediante le sonde perforate per le ricerche petrolifere, confermano la validita della formola di Schweydar, la prima delle [3], anche per le relativamente piccole profondita di interesse per le ricerche mineraric.

Nella frg. 1 riportiamo i valori, determinati dallo scrivente, della densità dei terreni attraversati dalla sonda N. 3 di Genzano, nella

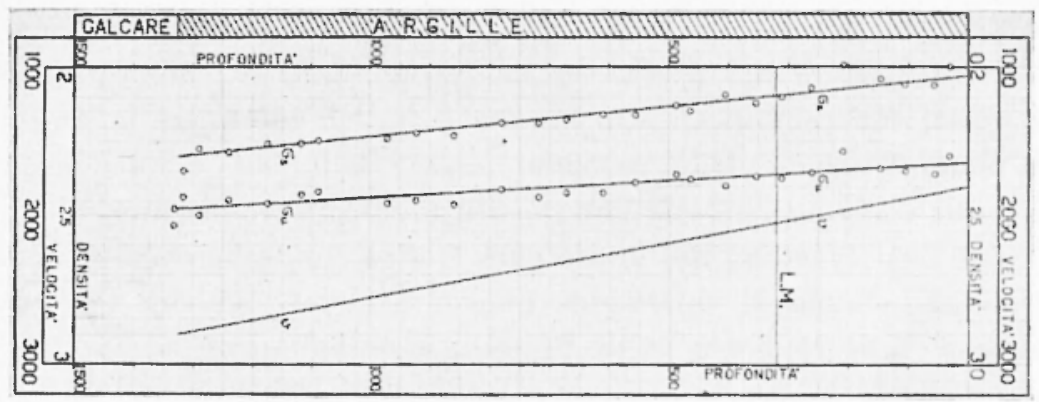

Fig. 1

valle dell Bradano, perforala nel 1941 dall'Azienda Generale Italiana Petroli: la curva contraddistinta da $\sigma_{u}$ dà $i$ valori relativi ai terreni saturi dell'acqua di strato e quella contraddistinta da $\sigma_{B}$ i ralori relativi agli stessi terreni seccati in corrente d'aria secca a $60^{\circ}$ Cent. Le differenze fra le due densiti sono evidentemente i valori della porosita. I valori di $\sigma_{0}$ e li \& sono rispettivamente di 2,03 e 0,00010 per la curva $\sigma_{*}$ e di 2,32 e 0,00005 per la curva $\sigma_{u}$ I valori di $\beta$ risultano sensibilmente nulli.

F notevole il fatto che la densila assoluta $\sigma_{a}$, ossia la densila riferita al volume ridotto della porosità,

$$
\sigma_{\mathrm{a}}=\frac{\sigma_{\mathrm{s}}}{1-\left(\sigma_{\mathrm{u}}-\sigma_{\mathrm{s}}\right)},
$$


risulti sensibilmente costante, uguale a 2,85 , indipendente dalla profondità e, di conseguenza, che l'aumento delle densitat $\sigma_{\mathbf{a}}$ e $\sigma_{\mathrm{u}}$ colla profondità sia dovuto pressoché eschusivamente alla diminuzione della porositì.

Raramente avverrà di trovare una pila di terreni omogenei per oltre 1300 metri di potenza, come nell'esempio riportato nella figura; in generale si troveranno delle curve della densità assai meno regolari: si noterà sempre però che per una stessa specie di roccia la densità sarà maggiore per le maggiori profondita e che mediamente si avrà un aumento della densitì colla profonditì secondo una legge del tipo della frima delle [3]. Come si sa, proprio sull'aumento della densità colla profondità e col tempo sono basate le possiluilita d'impiego dei metodi gravimetrici di rilievo, assai diffusi specialmente nella prospezione petrolifera.

La determinazione diretta del modulo di elasticita $E$ dei terreni è in generale molto difficile, se non impossibile, sia perché i terreni stessi si presentano d'ordinario con scarsa consistenza, sia perché molto laboriosa riesce la riproduzione in laboratorio delle condizioni di sollecitazione delle rocce in posto, sia perché è ancora incognito l'effetto combinato della pressione, della temperatura e del tempo d'azione. Si tende quindi, in generale, a determinare le costanti d'elasticità dei terreni per via indiretta. Uno dei metodi migliori di determinazione, usato principalmente dagli studiosi di fisica terrestre, $i$ quello di applicare la [2] misurando la $v$ e calcolando $r$, col procedimento contrario di quello che ora noi vorremmo applicare (\%).

Nella fig. 1 riportiamo anche l'andamento della velocita $v$ determinato mediante rilievi sismici a rifrazione e col metodo delle frecce dei rilieri a rillessione $\left({ }^{t}\right)$, dei terreni in corrispondenza alla stessa sonda n. 3 di Genzano. Tale andamento può essere rappresentato con sufficiente approssimazione dall'equazione

$$
v=v_{o}(1+m z),
$$

ove $v_{n}=1800 \mathrm{~m} / \mathrm{s}, m=0,00042 \mathrm{e} z$ in metri.

Note cosi le equazioni della densitì e della velocita possiamo determinare quella del modulo di elasticiti

$$
E=E_{\mathrm{o}}\left(1+\alpha^{\prime} z+\beta^{2} z^{2}+\gamma^{\prime} z^{3}\right),
$$

ove $E_{\mathrm{o}}=0,575 \cdot 10^{-11} \quad, \quad \alpha^{\prime}=144 \cdot 10^{-\hat{5}} \quad, \quad \beta^{\prime}=6,8 \cdot 10^{-8} \quad \gamma^{\prime}=11 \cdot 10^{-15}$ :

un'espressione che diviene uguale alla seconda della [3] quando si 
trascuri l'ultimo termine, il quale non arriva a superare il centesimo neanche per le maggiori profondita che interessano le ricerche minerarie.

2. Formule della velocità di trasmissione delle onde. -- Le espressioni [4] e [6] pososno rappresentare assai bene gli andamenti della velocití colla profondita quali risultano dalle misure dirette. Esse però presentano il grande inconveniente di condurre a dei calcoli eccessivamente lahoriosi nell'apprestamento dei prontuari di determinazione degli elementi necessari per i rilievi sismici a riflessione.

Per la pratica sono perciò preferibili le seguenti formole, le quali possono nei vari casi rappresentare altretlanto hene gli andamenti dei valori osservati,

$$
\begin{aligned}
& v=v_{0}(1+m z), \\
& \left.v=v_{0}\right\rceil \overline{1+n^{\prime} z}, \\
& v=v_{0}\lceil\overline{1+q z}, \\
& v=v_{0}\left[1+r\left(1-e^{-s z}\right)\right],
\end{aligned}
$$

la prima proposta indipendentenente da Ewing e Leet e da Caloi ( $\left.{ }^{5}\right)_{T}$ la seconda da V. Charles-E. Houston (") e la quarta da Solaini ("). La terza viene proposta ora dallo scrivente. Al solito $m, n^{\prime}, q, r, s$ sono delle costanti da determinarsi sperimentalmente.

Per i calcoli numerici in generale si usano, invece delle formole

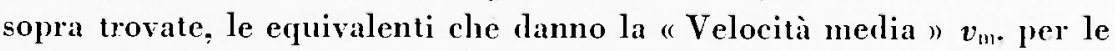
profondità da 0 a z per raggri verticali. Se poniamo in generale.

$$
v=v_{\mathrm{o}}(z) . \quad v_{\mathrm{m}}=v_{\mathrm{o}} \eta_{1}(z),
$$

risultano le relazioni

$$
\begin{gathered}
\psi(z)=\frac{z}{\int_{0} \frac{z d z}{\omega(z)}}, \\
\psi(z)=\frac{[\psi(z)]^{2}}{\psi(z)-\frac{d \psi(z)}{d z} z} .
\end{gathered}
$$

Otteniamo allora dalle $[7],[8],[9]$ e $[10]$, rispetivamente, 


$$
\begin{aligned}
& v_{\mathrm{m}}=v_{\mathrm{o}} \frac{m z}{\log (1+m z)}, \\
& v_{\mathrm{m}}=v_{\mathrm{o}} \frac{1}{2} \frac{u^{\prime} z}{\sqrt{1+n^{\prime} z}-1}, \\
& v_{\mathrm{m}}=v_{\mathrm{o}} \frac{3}{4} \frac{q z}{(1+q z)^{3 / 4}-1}, \\
& v_{\mathrm{m}}=v_{\mathrm{o}} \frac{\mathrm{sz}(1+r)}{s z+\log \left[1+r\left(1-e^{-s z}\right)\right]} .
\end{aligned}
$$

Nella fig. 2 riportiamo, colla curva $\sigma^{\prime}$ segrnata con linea grrossa piena, i valori medi della velocita media di alcune centinaia di determinazioni eseguite su terreni per età e per costituzione litologica simili a quelli presenti nella media valle Padana, almeno per le profondita fino a $3000 \div 4000$ metri; e con circoletti i valori determinati direttamente nella stessa valle Padana con apposite registrazioni esegruite in pozzi profondi 1 \%. Come si vede, le differenze fra tali punti e quelli corrispondenti della curva non superano mediamente il 10 per cento.

Nella stessa fig. 2 ahbiamo segnato anche le curve 2', 3', 4' e 5' ottenute rispettivamente dalle [12], [13], [14] e |15| coi valori delle costanti e di $v_{\mathrm{o}}$ più opportuni perché le stesse curve riuscissero il più possibile prossime alla 6' d'osservazione. La curva contraddistinta col numero l'è la corrispondente alla formola

$$
v_{\mathrm{m}}=v_{\mathrm{o}} \sqrt{1+n z}
$$

largamente applicata in Italia, la quale però comporta delle complicazioni notevolissime nel calcolo dei tempi di trasmissione e nelle equazioni dei raggi: la formola corrispondente per la velocita $v$, che per brevità chiameremo con "Velocità locale ", ̀̀

$$
v=v_{0} \frac{(1+n z)^{3},}{1+1 / n z} .
$$

Abhiamo adottato per la costruzione della curva 2' i valori $v_{0}=$ $=1800 \mathrm{~m} / \mathrm{s}$ ed $m=0,00033 \mathrm{l} / \mathrm{m}$, per la curva $3^{\prime} v_{0}=1800 \mathrm{~m} / \mathrm{s}$ ed $n^{\prime}=0,00086 \mathrm{l} / \mathrm{m}$, per la curva $4^{\prime} v_{\mathrm{o}}=1700 \mathrm{~m} / \mathrm{s}$ e $q=0,00451 / \mathrm{m} \mathrm{e}$ per la curva 5' $v_{0}=1675 \mathrm{~m} / \mathrm{s}, r=1,15$ ed $s=0,000651 / \mathrm{m}$, profondità z in metri.

Vediamo che la curva 4' è quella che più si approssima alla 6' osservata e che la 2' è quella che si approssima meno. 


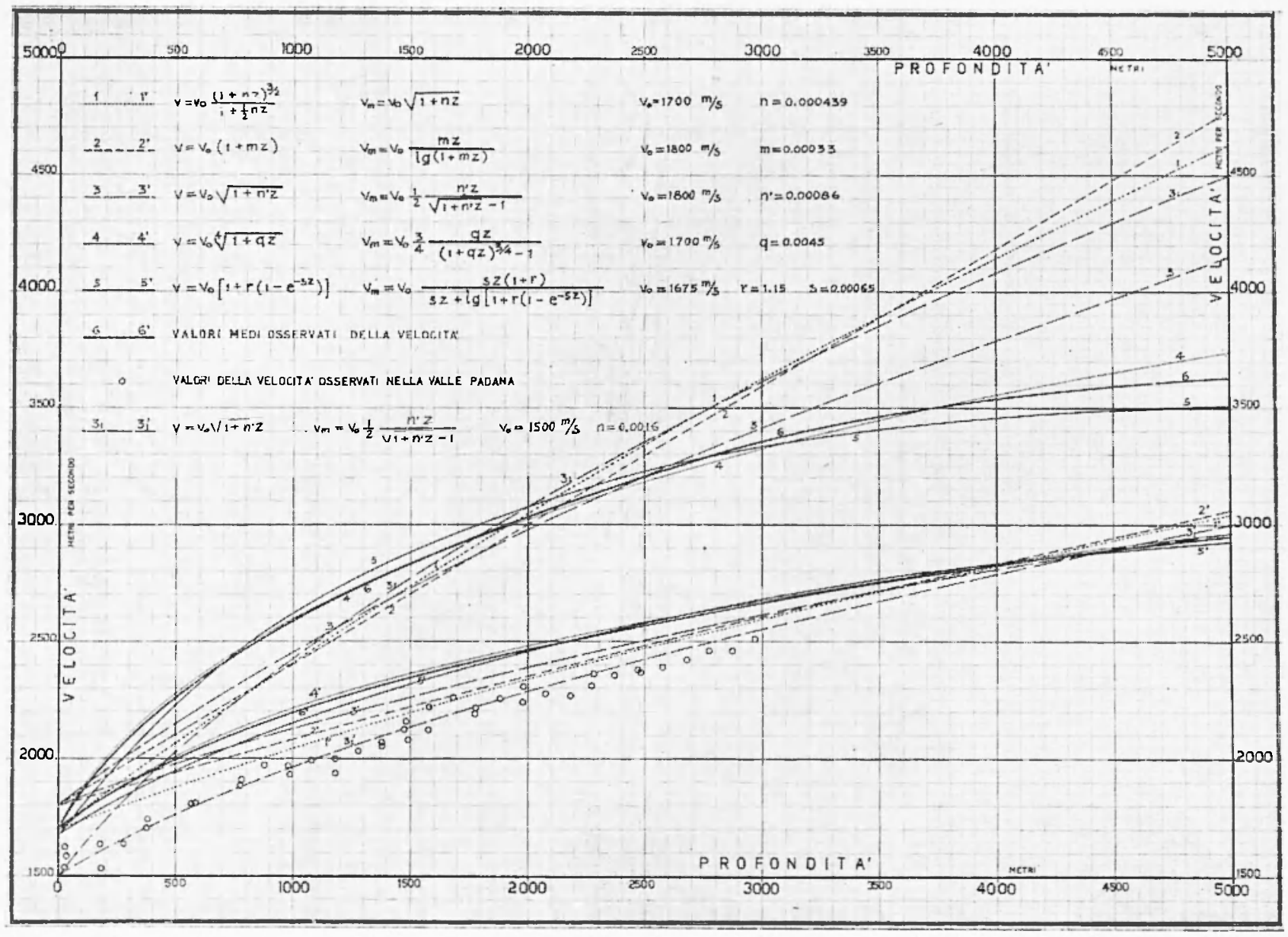

Fig. 2 
Per la costruzione della curva l' si sono adottati gli stessi valori per $v_{0}$ e per $n$ coi quali la [16] venne applicata praticamente nella valle Padana $v_{o}=1700 \mathrm{~m} / \mathrm{s}$ ed $n=0,0004391 / \mathrm{m}$.

Le curve contraddistinte coi numeri $1,2,3,4,5$ e 6 danno rispettivamente $\mathrm{i}$ valori delle velocità locali corrispondenti a quelli delle velocità medie delle curve 1', 2', 3', 4', 5' e 6', per gli stessi valori di $v_{0}$ e delle altre costanti.

Mentre per i calcoli dei valori delle curve $1,2,3,4$ e 5 si sono utilizzate le [17], [7], [8], [9] e [10] rispettivamente, per quelli dei valori della curva 6 si è utilizzata direttamente la seconda delle [11] sostituendo a $\psi(z)$ le ordinate di $\sigma^{\prime}$ e alle derivate la tangente trigonometrica della tangente alla stessa $\sigma^{\prime}$ nei punti corrispondenti.

Vediamo che anche considerando le velocità locali la formola [9] conduce a dei valori molto prossimi a quelli ricavati dalle osservazioni dirette mentre la [7] conduce ai valori in media meno prossimi.

Vediamo pure che le curve 1 e 2 aumentano assai rapidamente e che per profondità di oltre 5000 metri raggiungono dei valori della velocità troppo alti. Quando si incorre in un inconveniente del genere si può adottare l'artificio di ammettere una leggge diversa della velocità per le magrgiori profondità, a partire per esempio dalla profondità di 5000 metri. Al passaggio da una legge all'altra bisogna curare evidentemente perché vi sia raccordo, onde evitare la necessità di ammettere l'esistenza di variazioni repentine delle caratteristiche elastiche dei terreni, in generale per nulla giustificate.

Adottando due formole per la velocità, una valevole per le profondità da 0 a $z_{1}$ e indicando con $v_{0} \psi_{1}(z)$ la relocità media che si ottiene colla prima formola e con $v_{0} \psi_{2}(z)$ quella che si ottiene colla seconda determiniamo la velocità media $\left.v_{0}\right\}^{\prime}(z)$ valevole per $z>z_{1}$ mediante la

$$
v_{0} \psi^{\prime}(z)=v_{0}-\frac{z}{\frac{z_{1}}{\psi_{1}\left(z_{1}\right)}-\frac{z_{1}}{\psi_{2}\left(z_{1}\right)}+\frac{z}{\psi_{2}(z)}}
$$

Se per le formole [8] e [13] adottiamo i valori $v_{0}=1500 \mathrm{~m} / \mathrm{s}$ ed $n^{\prime}=0,0016 \mathrm{l} / \mathrm{m}$ otteniamo le curve $3_{1}$ e $3^{\prime}{ }_{1}$ in ottimo accordo coi valori osservati della media valle Padana (tenuto conto anche dei valori ottenuti colle più recenti determinazioni in nostro possesso, che non siamo autorizzati a pubblicare). 
3. Influenza delle eterogeneità e dell'anisotropia dei terreni. --- In effetto nessuma formola della velocita può rappresentare rigorosamente l'andamento della velocitì stessa colla profonditi in quanlo il sottosuolo non è costituito da terreni omogenei e uniformi ma invece dalla successione di molti strati, aventi caratteristiche fisiche e litologiche diverse, lali da provocare rapide variazioni dei valori della velocila. Se così non fosse, come si sa, non potrebbero aver luogo le riflessioni dell'eneroria sismica e quindi i rilievi sismici, che appunto si basano sulla misura dei tempi delle onde riflesse, non potrebhero dare risultati utili. Nella pratica runque noi dobbiamo accontentarci di formole che diano l'andamento medio: quando queste si approssimano ai valori reali, per tutte le profonditi, con differenze minori del $10 \div 15$ per cento già si ritengono buone, ottime quando le differenze risultamo del $5 \div 10$ per cento.

Il fallo che i terreni del sottosuolo siano disposti in strati comporta un'anisotropia per rispelto alle caratteristiche elastiche e quindi per rispetto alla relociti $v$ di trasmissione delle onde.

Gutenberg (") trova che in generale la velocita di tramissione delle onde nel senso della stratificazione risulta sensililmente maggiore di quella nel senso normale e che la differenza può raggrungere anche valori dell'ordine del 20 per cento.

Giò posto, appare evidente come le determinazioni della velociti fatte mediante i cosidrletti "Profili di rifrazione", con raggi che di solito si trasmettono nel senso longitudinale degli strati, riescano praticamente molto grossolane agli effetti dei rilievi sismici a riflessione, i quali viceversa sono hasati sulla misura dei tempi di ragri pressoché perpendicolari agli strati riflettenti. Ugualmente appare evidente come in pratica convenga escludere per i rilicvi stessi quelle disposizioni dei sismografi e dei pozzetti di scoppio che utilizzano raggi diversamente inclinati rispetto agli strati riflettenti $\left({ }^{9}\right)$, e quindi diversamente influenzati dall'anisotropia.

E infine da considerare cle in generale per effetto dellanisotropia dei terreni le lince hrachistocrone, ossia i raggi sismici, non risultano perpendicolari alle superfici d'onda e gli stessi raggi, nella riflessione e nella rifrazione, si comportano in modo alquanto diverso di quello voluto dalle leggri classiche.

4. Andamenti delle isotuche. -.. In generale gli strati costiluenti il sottosuolo furono deposti in origine secondo griaciture orizzontali o sulb- 
orizzontali: tali strati quindi subirono le prime compressioni, dovute alla graviti, nel senso ortogonale, le quali provocarono nello stesso senso un incremento della costipazione e di conseguenza della densità e della velocità $v$ di trasmissione delle onde. Questo incremento, per quanto abliamo detto, è da ritenersi funzione del carico e del tempo d'azione dello stesso.

Se successivamente gli stessi strati, perdurando oppure no la deposizione dei sedimenti, fossero stati piegati il carico avreble agito in senso obliquo rispetto alla stratificazione e di conseguenza si arreblee avuto col passar del tempo un incremento non più ortogronale ma secondo una dirczione intermedia fra l'ortogonale e la verticale, più vicina all'una o all'altra a seconda del rapporto dei carichi e dei tempi d'azione prima e dopo il piegamento.

Le superfici luogro dei punti di uguale velocità $v$, che per comorlità chiameremo con "Superfici isotache", le quali erano pressoché parallele agli strati quando questi erano orizzontali, dopo il piegamento si disposcro sempre più oblique al passare del tempo.

Se il piegamento fosse arvenuto repentinamente le isotache, parallele agli strati, avreblyero riprodotto esattamente l'andamento delle pieghe e quindi si arrehbero avute delle velocita $v$ corrispondenti agrli stessi livelli molto diversi da posto a posto. Ma in generale $i$ piegamenti venmero prodotti lentamente e gradualmente in modo da permettere l'esplicazione di un'efficace azione degli clementi livellatori che di solito insorgono coi piegamenti stessi, quali l'erosione superficiale ad opera degrli agenti atmosferici e l'erosione sottomarina, l'intensità della sedimentazione diversa in corrispondenza alle diverse quole dei fondi marini, in generale crescente al crescere della profondita delle acque, le forze d'equilibramento isostatico, ecc. Tali elementi agiscono tuti nel senso di diminuire grli squilibri delle pressioni corrispondenti alle zone più o meno sollevate dai piegamenti e quindi nel senso di diminuire i divari fra $i$ valori delle velocita delle onde sismiche corrispondenti alle stesse zone, per i punti di ugual quota.

D'altra parte, poiché l'incremento della velocità col tempo non può che diminuire col tempo stesso, naturalmente a parità delle altre condizioni di pressione, temperatura, ecc. cosi in generale averrà che anche al crescere dell'età dei terreni i divari dei valori della velocita

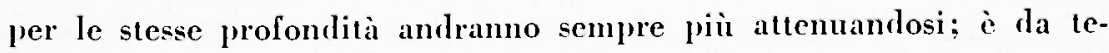
nere presente però che i livari dovuti al fatto che coi piegamenti 
furono portati a quote diverse terreni di diverse caratteristiche litologiche permarranno sempre e che quindi anche per un'etit grandissima dei terreni stessi questi daranno sempre luogo a delle superfici isotache con andamento non orizzontale sebbene discorde da quello degli strati.

In pratica risulta molto difficile determinare con esattezza l'andamento delle superfici isotache in corrispondenza alle pieghe del sottosuolo, in quanto $\mathrm{i}$ sondaggi di ricerca vengono di solito limitati alle zone apicali e conseguentemente ai fianchi e sulle sinclinali le misure delle velocita di trasmissione delle onde devono essere fatte

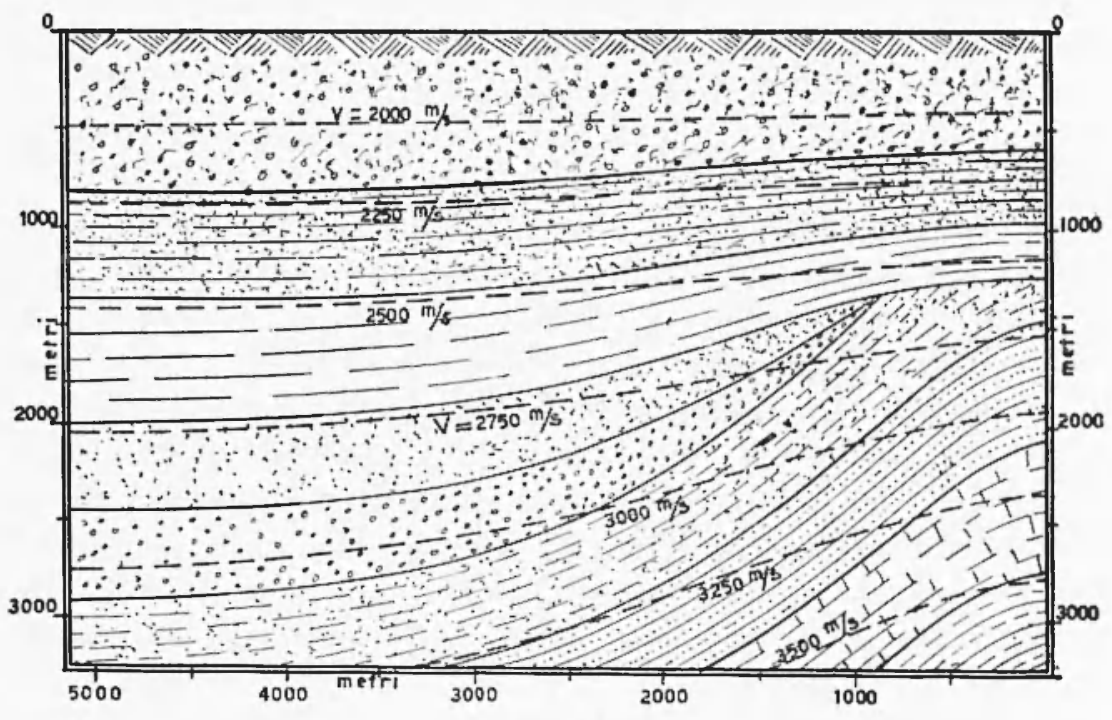

Fig. 3

coi metodi delle frecce, i quali, come si sa, risentono dell'influenza delle curvature e delle inclinazioni delle superfici riflettenti, e risultano sempre di precisione molto scarsa. È da tenere presente che non è consigliabile porre a confronto valori relativi a punti fra loro molto lontani, i quali possono risultare corrispondenti a facies sensibilmente diverse dei terreni.

Riferendoci però a quanto abbiamo detto più sopra e a quanto risulta dalla nostra esperienza e dalla letteratura geofisica nota, possiamo schematizzare come nella fig. 3 gli andamenti delle isotache per le strutture d'interesse minerario, quali si presentano nella media valle Padana, tipo Caviaga, Ripalta, Soresina, Bordolano, Corte- 
magrgiore, Pontenure, Turro, ecc. Queste strutture, come si sa, sono caratterizzate da un nocciolo centrale miocenico, intensamente piegato, con strati generalmente porosi, e da una copertura pliocenicaquaternaria di spessore fino a $2000 \div 3000$ metri, angolarmente discordante sul nocciolo, in generale argillosa alla base e molto porosa alla sommitì.

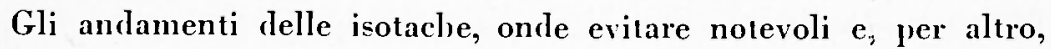
inutili complicazioni dei calcoli, sono state tracciate nell'ipotesi che sia nulla l'influenza sui valori della velocità della diversa costituzione litologica degli strati e quindi considerando semplicemente le influenze dell'azione del tempo e della particolare tettonica.

Vediamo che in effetto risulta riprodotto nelle isotache l'andamento degli strati piegati, sebbene con dislivelli molto attenuati. Ugualmente averreblue evidentemente per le faglie e le altre discontinuità degli strati che pure occorrono sovente nelle strutture della media valle Padana. Vediamo anche che al crescere delle profondita le isotaclue aumentano $i$ dislivelli fra $i$ punti corrispondenti alle sinclinali e alle anticlinali; è evidente però, per quanto abbiamo detto, che tale andamento dovrà invertirsi da una certa profondità in poi.

5. Andamenti delle brachistocrone. - Per tener conto delle deformazioni delle superfici isotache conseguenti alle vicende geologiche-tettoniche subite dai terreni in studio dovremmo evidentemente adottare delle formole in cui figurassero anche le coordinate orizzontali. In pratica però, per evitare le notevoli complicazioni dei calcoli conseguenti, si preferisce in generale adottare una delle formole della velocità, funzione soltanto della profondita $z$, che abbiamo visto al paragrafo precedente, e applicare alcuni facili accorgimenti atti a ridurre gli errori relativi.

Se adottiamo delle formole che diano i valori esatti della velocità in corrispondenza all'apice delle strutture si ottengono in generale delle profondita esagerate per le sinclinali e, viceversa, se si adottano dei valori esatti per le sinclinali si hanno delle profondità troppo piccole per le anticlinali: in entrambi $i$ casi si ha un aumento dei dislivelli fra sinclinale e anticlinale. Uguali risultati si hanno per valori intermedi della velocita. Dovendo, come si vele, provocare in ogni caso delle deformazioni degli andamenti conviene in pratica cercare di adottare delle formole che siano esatte per le zone apicali delle 
strutture, le quali nomalmente sono di magrior interesse pre le ricerche.

Le lince per l'origine dei sismi normali in ogni punto alle superfici isotache sono lince lorachistocrone. Se le isotache sono piane e parallele tali linee sono rette; in questo caso l'equazione dei raggi sismici si determina facilmente adottando come asse delle z tali rette. E facile vedere però che cosi operando i raggi riflessi dagli strati risultano determinati con delle (Aperture) maggiori di quelle dei ragri che si otterrebbero ponendo verticale l'asse delle $z$ e supponendo orizzontali le isotache.

Indichiamo con "Aperture " grli angoli ‘ formati colla verticale dalle congiungenti i punti dei ragrgi collorigine dei sismi.

Nel caso limite delle isotache parallele agli strati, possibile come abhiamo detto nel caso dei sollevamenti repentini, si avreblero dei raggi pressoché rellilinei, di apertura massima allincirea uguale all’inclinazione a delle superfici rifletenti.

Per la pratica è da osservare che se si ammellono dei rạgi con aperture troppo piccole le strutture che risultano dai rilievi appaiono discontinue in corrispondenza ai punti con curvatura rapidamente variabile e in generale troppo larghe ai fanchi e che, viceversa, se si ammettono dei raggi con aperture eccesive le strutture applationo troppo strette e, nei punti di rapida variazione della curvatura, con sovapposizioni di clementi e con andamento incerto.

Nel passato si sono larramente usati pei rilievi pratici sia ragrgi con aperture abbondanti, quali sono quelle dei raggi rettilinei, sia raggi con aperture scarse, quali quelle teoriche conseguenti alle formole adottate per le velociti. Lo scrivente, per conto suo, ha aruto modo di applieare, per il rilievo delle strutture della media valle Padana, i ragrgi che risultano dalla formola [16] della velocita ponendo per le superfici d'ondal l'equazione

$$
\varrho=\frac{\tau}{2} v_{\mathrm{o}} \sqrt{1+n z},
$$

ove o sia la distanza dei sismi dall'oriqine, $\frac{r}{2}$ il tempo trascorso dall'istante di scoppio, $v_{0}=1700 \mathrm{~m} / \mathrm{s}$ ed $n=0,0004391 / \mathrm{m}$. nelle due ipotesi: dei raggi rettilinei, fino a tutto il 1949 , c dei raggi curvilinei in ogni punto ortoggonali alle superfici d'onda, dopo il 1949. La forma dei raggi che si ottiene ponendo quest'ultima ipotesi i pressoché uguale 
a quella teorica per terreni omogenei ed jsotropi, almeno per le profonditi minori di $2500 \div 3000$ metri, che più interessano per la pratica.

Adotando delle formole approssimate per il calcolo delle equazioni dei ragroxi e dei tempi i inevitabile che si debbano introdurre anclue delle contraddizioni e delle incsattezze. Per il caso della !19] e della condizione di ortogonaliti sequente é però da tenere presente ch in ogni caso, adottando la lormola [16], avremmo dovuto ricorrere a espressioni approssimate, dato che la stessa formola, come gria abliamo avertito, non permette la determinazione rigorosa delle stesse equazioni dei rạgrai e dei tempi. Si deve considerare che arlottando dei rag̣̣i basati sulla stessa formola [16] si sono resi facilmente confrontabili i lati dei rilievi esegruti prima e dopo il 19.19.

Dalla nostra esperienza e, in generale, da quella di tutti i geofisici che hanno esegruito dei rilievi sul terreno risulta chiaramente dimostrato che per ricostruire esattamente la forma delle strutture i necessario adottare dei ragri arenti aperture intermedie fra quelle che si hanno coi ragrgi rettilinei e quelle dei rageri che si ottengrono teoricamente applicando i principi di Fermat o della brachistocrona di Lagrange.

Quale sia pero la forma più opportuma da adottare per i raggi solo volta per volta si potrì stabilire, a rilievo ultimato, in base alle particolari condizioni greologriche-tettoniche riscontrate e alla presenza delle discontinuiti o delle sovrapposizioni degli elementi in corrispondenza ai punti di rapida variazione della curvatura di cui sopra abbiamo detto.

Evidentemente, per strutture del tipo di quella rappresentata nella figr. 3, le forme dei rag̣gi devono essere vieppiù "Aperte ", ossia con aperture sempre magreriori, al diminuire dello spessore dei terreni discordanti di copertura e dell'etí di questi, come pure al crescere della differcnza d'eti fra i terreni di copertura e quelli del nocciolo centriite intensamente piegato.

L'anisotropia dei terreni, dato che in generale gli strati superficiali sono meno pendenti di quelli profondi, agrisce pure sui ragrgi nel senso di renderli piì aperti, naturalmente in modo più o meno sensibile a seconda delle differenze delle velociti nella direzione della stratificazione $e$ in quella perpendicolare.

Nella fig. 1 riportiamo le curve dei ragri sismici corrispondenti agli stessi tempi $r$, di 1500,2500 e 3500 us, per superfici riflettenti della stessa inclinazione di $30^{\prime \prime}$ (pressapoco alle profondita di 1509, 
3000 e $5000 \mathrm{~m}$ rispettivamente) relative alle formole della velocità [7], [8], [9] e [10] già considerate per la fig. 2, a confronto con quelle relative alla [19] raggi curvilinei, la N. 1, e raggi rettilinei, la N. 1". I numeri distintivi delle altre curve sono gli stessi usati per le curve della fig. 2; evidentemente con $z$ sono indicate le profondità e con $x$ le distanze orizzontali misurate dall'origine dei sismi.

Vediamo da tale figura che le aperture dei raggi risultano molto differenti per le diverse formole, alle grandi profondita, pur essendo tutti relativi a superfici riflettenti della stessa inclinazione di $30^{\circ}$. Si può facilmente comprendere da tale figura come le forme delle strutture possano riuscire molto diverse adottando i raggi rettilinei $1^{\prime \prime}$ oppure i curvilinei e come per le maggiori profondità si possano avere delle sensibili differenze anche arlottando luna piuttosto che l'altra specie di raggi curvilinei.

Le possibilità di controllo della forma delle strutture, nella valle padana, non vanno oltre i $2000 \div 3000$ metri di profondita, percio noi in effetto non abbiamo alcun elemen to per stabilire quale possa essere la forma dei raggi più appropriata da adottare per le maggiori profonditi. Può darsi che per queste maggiori profondità risulıino ad. dirittura adatti i raggi che si ottengono teoricamente, per esempio quelli 4 o 5 relativi alle formole [9] e [10], oppure i ragroi rettilinei.

Per le minori profondità tutti i raggi curvilinei comportano pressapoco le stesse aperture ed è a queste che dobbiamo riferirci quando diciamo che l'esperienza consiglia di adottare dei raggi con aperture intermedie fra quelle dei raggi rettilinei (che comportano delle strutture troppo strette ai fianchi) e quelle dei raggi teorici curvilinei (che viceversa comportano delle strutture troppo larghe ai franchi).

Nella fig. 4 riportiamo anche, in una tabella, $i$ valori degli angoli $a_{0}$ che $i$ raggi formano colla verticale all'origine dei sismi, $i$ quali sono caratteristici dei diversi ragri e possono dare un'idea della curvatura media dei raggi stessi. L'angolo $\alpha=30^{\prime \prime}$ è il complemento delle inclinazioni dei raggi in corrispondenza ai punti di riflessione.

6. Calcolo delle superfici riflettenti. - Un procedimento, che possiamo ritenere generale, per la determinazione approssimata dei ragri da adottare per il calcolo delle superfici riflettenti è il seguente. Supposto di avere noto l'andamento delle superfici isotache della zona da rilevare determiniamo la retta normale media alle stesse superfici per ogni punto $P$ ove si sono eseguite le misure: sia $\delta$ l'angolo for- 


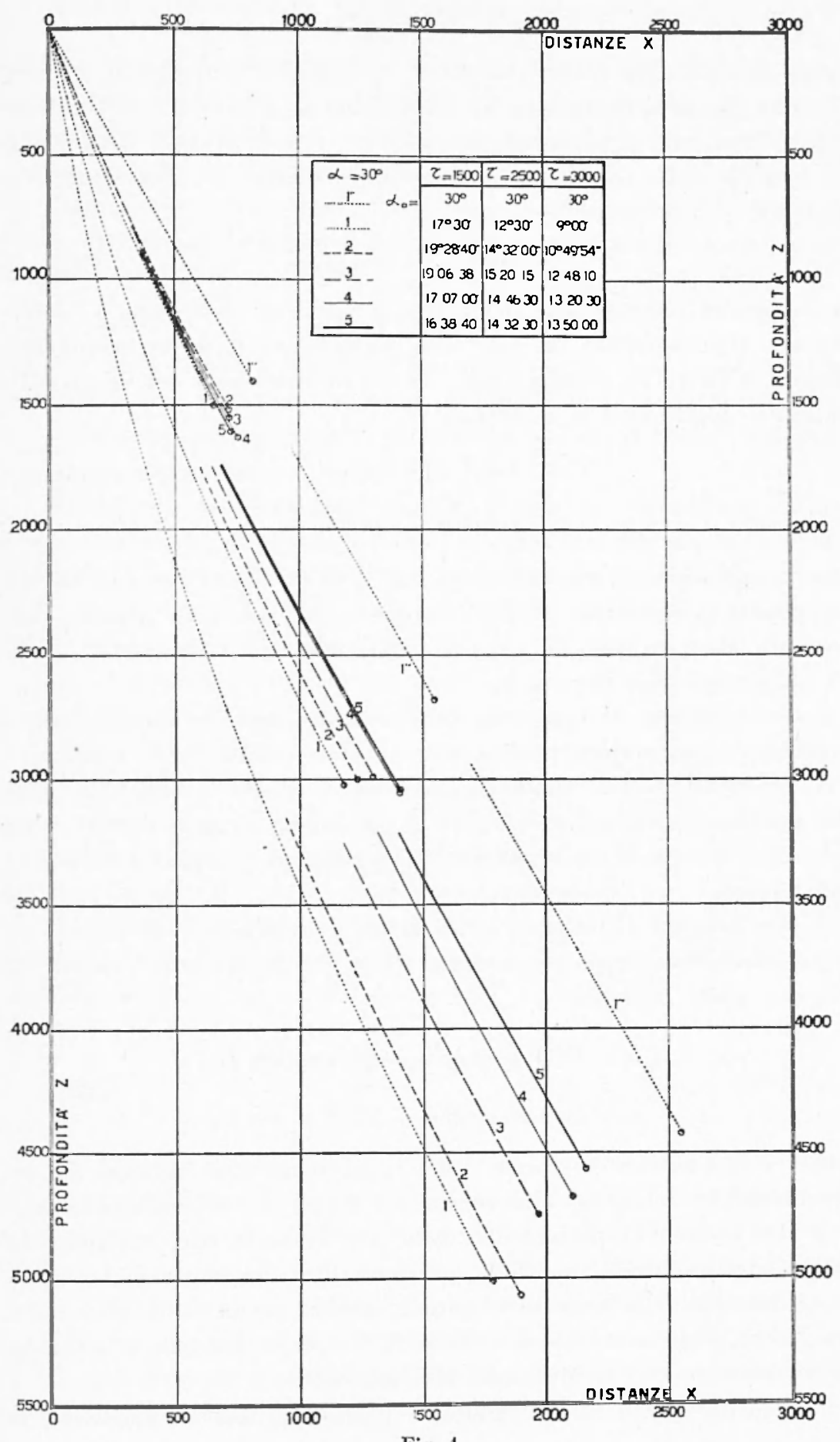

Fig. 4 
mato da tale retta colla verticale. L'inclinazione a delle superfici riflettenti si puó, in prima approssimazione, supporre indipendente dalla forma dei ragreri adottata quando i calcoli relativi sono hasali su formole della velocita abhastanza approssimate. Se allora indichiamo con

$$
x_{\mathrm{r}}=\mathrm{X}(\tau) \quad, \quad z_{\mathrm{r}}=Z(\tau)
$$

le equazioni parametriche dei raggi, in funzione dei tempi r, determinate rigorosamente in batse alla formola adottata della velocita, possiamo porre in generale per i punti di riflessione dei raggi delle superfici degli strati le equazioni

$$
\begin{aligned}
& x=\mathrm{X}(\tau)+\boldsymbol{U}^{\prime}(\alpha, \tau), \\
& z=Z(\tau)+\Phi^{\prime}(\alpha, \tau),
\end{aligned}
$$

ove $r$ siano i tempi misurati e $\Phi(\alpha, \tau)$ e $\Phi^{\prime}(\alpha, \tau)$ due hunzioni da determinarsi in relazione ai motivi tettonici particolari da rilevare, alla formola adottata per la velocita, all'angolo o risultante, al grado di anisotropia dei terreni, ecc.

Praticamente dunque con tale procedimento le inclinazioni " verrebhero determinate mediante i prontuari ordinari e le coordinate $x$ e $z$ verrehbero determinate aggiungendo a quelle $x_{r}$ e $z_{1}$, date pure dai prontuari ordinari, dei fattori di correzione da determinarsi caso per caso a seconda delle particolari condizioni geologiche-tettoniche dei terreni.

Per la pratica possiamo tener conto di tali fattori di correzione e nel medesimo tempo tener conto delle variazioni della velocità da posto a posto ponendo

$$
\begin{aligned}
& x=X(\tau) p \cos f \alpha+Z(\tau) p \operatorname{sen} f \alpha, \\
& z=Z(\tau) p \cos f u+X(\tau) p \operatorname{sen} f u,
\end{aligned}
$$

ove $f$ è il "Fattore di forma " dei raggi, ossia una costante da determinarsi in relazione alla forma dei raggi che si vuole adottare, e $p$ il "Fattore di posizione ), ossia una costante che, variando da punto a punto della superficie, permetta di tener conto delle variazioni laterali della velocità e quindi, anche, permetta di eliminare, fra l'altro, l'inconveniente dei dislivelli esagerati fra base e sommiti delle strutture di cui più sopra abhiamo detto.

E facile vedere che in effetto, a parte il fattore di posizione, le 
[22] non sono altro che le [20] riferite ad un sistema di assi cartesiani rotato dell'angolo $\delta$, nell'ipotesi che questo sia uguale ad una frazione $f$ dell'inclinazione $a$ delle superfici riflettenti e quindi variabile per ornuma delle stesse superfici.

Naturalmente le costanti $f$ e p sono interdipendenti e debhono essere determinate contemporaneamente: approssimativamente dovrà risultare

$$
p=\cos f\left(\iota_{m},\right.
$$

ove con $\alpha_{m}$ ̀̀ indicata l'inclinazione media degli strati compresi fra la verticale per il punto considerato e quella per l'apice delia struttura, in corrispondenza al quale le formole adottate della velocita sono o si suppongono esatte.

I centri di curvalura delle superfici d'onda non coincidono, in generale, coll'origine dei sismi, quindi colla rotazione dell'angolo o del sistema degli assi di riferimento, di cui abbiamo detto, gli elementi non risultano più corrispondenti ai tempi $\tau$ d'osservazione, suposto di ritenere sempre valevole la legrge della velocita ammessa. Un inconveniente del grenere, e per di più molto agrgravato, si ha adottando il sistema semplice di agriungere alle distanze $x$ delle quantità percentuali delle stesse distanze. Eliminiamo evidentemente oğni inconveniente se supponiamo di rotare gli elementi in morlo che questi risultino posti sempre sulle stesse superfici d'onda, ossia di rotare grli stessi elementi intorno ai centri di curvatura delle superfici d'onda relative. Uguali risultati in pratica si raggiungono, per valori abbastanza piccoli di $\delta$, se supponiamo che $i$ centri di curvatura siano coincidenti colle intersezioni delle nomali alle superfici d'onda colla verticale per l'origine dei sismi e quindi poniamo

$$
\begin{aligned}
& x=p X(\tau)+p X(\tau) f \frac{u}{t g \alpha} \\
& z=p Z(\tau)-p X(\tau) f u .
\end{aligned}
$$

Per le solite strutture della media valle Parlana possiamo arl esempio porre: arlottando la formola [16] della velocita e la condizione di ortogronalita dei ragrai e delle superfici d'onda $f=4 / 30$ per le formole [22] ed $f=1 / 5$ per le [24], adottando la $|8|$ della velocità $f=0,09$ per le [22] ed $f=0,12$ per le [24] e arlottando la [9] della velocità $f=0,06$ per le $[22]$ ed $f=0,08$ per le $[24]$.

Il procerlimento sopra indicato per tener conto delle variazioni della velocità da posto a posto, mediante il fattore di posizione, è sicura- 
mente molto grossolano: in effetto però si troverà sempre che i dati sui valori della velocità di cui possiamo disporre sono tanto scarsi e tanto imprecisi da rendere superfluo ogni tentativo volto a migliorare in conseguenza le determinazioni delle profondità. In generale, quindi, non si potrà in pratica rhe adottare il valore 1 per il fattore di posizione.

Posto $p=1$ e fissato per $f$ il valore più appropriato alle condizioni geologiche-strutturali della zona di rilievo, si possono predisporre, mediante le [24] o le [22], i soliti grafici-prontuario per il calcolo delle coordinate $z$ ed $x$ dei punti di riflessione delle superfici riflettenti degli strati.

Quando, ad ogni modo, si adottasse per p un valore diverso dall'unita si moltiplicheranno per $p$ stesso i valori che risulteranno dagrli stessi grafici.

I calcoli dei dati necessari per costruire i grafici-prontuario riusciranno molto agevolati se già si disporrà di quelli relativi alle [20], alle quali si riducono le [22] e le [24] per $p=1$ ed $f=0$.

Naturalmente, fissati che siano i valori dei fattori di posizione $p$ e di forma $f$ si possono anche calcolare i valori delle correzioni conseguenti da apportare alle inclinazioni a delle superfici riflettenti determinate, mediante le equazioni generali [20], nell'ipotesi che fossero nulli gli stessi fattori $p$ ed $\int$. Tali correzioni, che indichiamo con ou, sono date dalla

$$
\delta \alpha=-\operatorname{tg} \alpha\left[p X(\tau) f \alpha \cdot \frac{1}{v} \frac{d v}{d z}-(p-1)\right] .
$$

ove $\frac{d v}{d z}$ non è altro che la pendenza della curva della velocità $v$ adottata, $1,2,3,4,5$ oppure 3 , della fig. 2 , in corrispondenza al valore di $z$ dato dalla seconda delle [20], e $v$ è il valore della velocità cor. rispondente allo stesso valore di $z$.

\section{RIASSUNTO}

Vengono esaminate alcune formole della velocità di trasmissione delle onde sismiche nel sottosuolo della media valle Padana, di cui l'autore ha particolare esperienza, e l'influenza dellanisotropia degli strati e delle loro vicende geologiche-tettoniche sulle caratteristichs geometriche delle curve brachistocrone. Viene quindi proposto un si- 
stema di calcolo delle superfici riflettenti che permette la ricostruzione, sufficientemente esatta per la pratica, delle strutture mediante $i$ dati ottenuti dagli ordinari rilievi sismici a riflessione.

\section{SUMMARY}

Some of the formulat relating to the seismical wave transmission speed in the underground of the Po Valley (this being the area the Author better knous by experience) have been investigated, as well as the influence of strata anisotropy and geological-tectonic history on geometric characteristics of brachystochronous curves. Author proposes hence a method which allows to obtain -- with a sufficient precision for practical purposes, and using data obtained through normal seismical reflection observations -- the recostruction of the various structures.

\section{BIBLIOGRAFIA}

(1) S. S. WEST: Dependence of seismic wave velocity apon depth and lithology. Geophysics, Vol. XV, N. 4, 1950.

- L. Y. FaUST: Seismic velocity as a function of depth and geologic time. Geoplyysies, Vol. XVI, N. 2, 1951.

(2) B. Gutenberc: The elastic constants in the interior of the Earth. Dal volume Internal Constitution of the Earth a cura del National Research Council degli U.S.A., 1951.

(3) D. S. Hugnes and J. H. Cross: Elastic uave velocities in rocks at high pressures and temperatures. Geophysics, Vol. XVI, N. 4, 1951.

(1) C. Costixi: Determinazione della velocitì di trasmissione delle onde sismiche nei rilievi a riflessioni. Atti del VII Congresso Nazionale del Metano e del Petrolio di Taormina dellaprile 1952.

(ii) Maunice Ewise and Dox LefT: Seismic propagation paths. A.I.M.M.E., 1932.

(6) V. Chart.fs E. Houston: Seismic paths, assuming a parabolic increase of velocity with deptl. Geoplysics, Vol. IV, N. 4, ottobre 1939.

- Pietro Caloo: Caralleristiche sismiche fondamentali dellEuropa Centrale. Boll. Soc. Sism. lt, 19-13.

(i) Lutg Solani: Corso di Geofisica Mineraria, Istituto di Geofisica applicata del Politecnico di Milano, 19.49.

(s) C.. Maxo: Misure nei pozzi delle velocità delle onde sisniche. Atti del VII Congresso Nazionale del Metano e del Petrolio di Taormina dell'aprile 1952.

(9) C. Coxtrivi: L'applicuzione dei rilievi sismici a riflessione continui. Metano, Anno VI, N. 1 e 2, 1952. 